This is the submitted version of the following article: 'Does Visual Spatial Awareness Require the Visual Awareness of Space?', forthcoming in Mind and Language. All citations should be to the published version.

\title{
Does Visual Spatial Awareness Require the Visual Awareness of Space?*
}

\author{
John Schwenkler
}

\begin{abstract}
Many philosophers have held that it is not possible to experience a spatial object, property, or relation except against the background of an intact awareness of a space that is somehow 'absolute'. This paper challenges that claim, by analyzing in detail the case of a brain-damaged subject whose visual experiences seem to have violated this condition: spatial objects and properties were present in his visual experience, but space itself was not. I go on to suggest that phenomenological argumentation can give us a kind of evidence about the nature of the mind even if this evidence is not absolutely incorrigible.
\end{abstract}

\section{The Apriority Thesis}

It is natural to think that the representation of space has a status in perceptual experience that is reasonably called 'a priori' in at least the following sense: it is not possible for the perceptual awareness of space to have been built up from a series of independent perceptions of spatial objects, properties, or relations, for perceptual experiences of the latter sort are possible only as modifications of the awareness of space itself. Kant famously advances such a claim in the Transcendental Aesthetic:

* I am especially grateful to John Campbell, Thane Naberhaus, Alva Noë, Bill Prinzmetal, Lynn Robertson, James Stazicker, Daniel Warren, and two anonymous referees with this journal for comments and discussions concerning this material.

Address for correspondence: Department of Philosophy, Mount St. Mary’s University, Emmitsburg, Maryland 21727 USA

E-mail: schwenkler@msmary.edu 
$\ldots$ in order that certain sensations be referred to something outside me (that is, to something in another region of space from that in which I find myself), and similarly in order that I may be able to represent them as outside and alongside one another, and accordingly as not only different but as in different places, the representation of space must be presupposed. The representation of space cannot, therefore, be empirically obtained from the relations of outer appearance. On the contrary, this outer experience is itself possible at all only through that representation. (Kant 1933, p. A23/B38)

The idea here is that perceptually representing the spatial distinctness of sensations necessarily requires the perception of a single space within which those sensations are represented as distinctly located; otherwise there can be no 'outer experience' of the sort in question. Let's call this the Apriority Thesis, or 'AT'. (Its details will be sharpened soon enough.) According to some commentators (e.g. Strawson 1966, p. 58; Allison 1983, p. 83; Guyer 1987, p. 346) the Apriority Thesis, thus articulated, runs the risk of being true only because it is tautological; but my purpose in this paper is to show how it can be brought into question on empirical grounds. I will also make some general remarks about the epistemology of phenomenological analysis. Before this, however, we will take some time getting clearer on what the thesis in question is supposed to say.

\section{Space, Spaces, and Objects}

One philosopher who has more recently attempted a defense of something like the Apriority 
Thesis is Quassim Cassam, who argues in The Possibility of Knowledge for what he calls the Spatial Perception Requirement:

(SPR) In order to perceive that something is the case and thereby to know that it is the case one must be capable of spatial perception. (Cassam 2007, p. 88)

According to Cassam the truth of SPR is a consequence of the fundamental status of primary qualities in the constitution of physical objects, i.e. the fact that what makes a physical object the kind of thing it is is its possession of properties like shape, location, solidity, and so on. For this reason, he argues, any perceptual episodes that can contribute to empirical knowledge of the physical world 'must somehow be connected to the ability to perceive [the] primary qualities' of things (2007, p. 120). If Cassam's argument succeeds, then it will have shown that a certain sort of spatial awareness is a condition of the possibility of perceptually-grounded knowledge.

But AT seems to claim something other than this. For according to Cassam the capacity for spatial perception that is a precondition on the perception of objects 'can either be understood as the capacity to perceive spatial properties or as the capacity to perceive space' (2007, p. 91). An Apriority Theorist, on the other hand, focuses precisely on a distinction that Cassam here glosses over: the precondition identified by Kant is a precondition on our ability to represent things 'as outside and alongside one another, and accordingly as not only different but as in different places', which he argues is impossible without already representing them as 'in space'. As Daniel Warren puts it, Kant believes that in order to represent spatial properties and relations 'a distinct representation, and thus, a distinct capacity is presupposed, namely a capacity to represent the spaces which objects occupy' (Warren 1998, p. 210). So even if an Apriority 
Theorist might agree with Cassam, he or she would add to SPR a further claim, namely that the possibility of spatial perception in Cassam's sense, i.e. the perception of spatial properties and relations, requires the capacity for the awareness of space itself. And this hypothesis is logically independent of Cassam's.

With this in mind, let's refer to the kind of perceptual awareness that Cassam is concerned to reveal as a condition of the possibility of empirical knowledge 'spatial perception' (or 'mere spatial perception'), reserving the title 'awareness of space' (or 'awareness of space in the strict sense') for the kind of spatial representation that is the focus of the Apriority Theorists. The paradigmatic examples of 'spatial perception' will be the perception of spatial objects, spatial properties, and spatial relations: all these forms of perception involve representing sensory elements 'as outside and alongside one another, and accordingly as not only different but as in different places'. By contrast, 'awareness of space in the strict sense' simply means the awareness of space: that is, awareness of the sort of thing that Newton (and Kant) supposed could be 'empty' and was somehow independent of the objects within it, and that according to the Apriority Theorist constitutes a kind of experiential framework within which all particular spatial things are necessarily represented as situated. If we read Kant's talk of 'the representation of space' as picking out this second notion, then clearly he is saying something other than Cassam is prepared to defend: on this understanding of AT, whether or not spatial perception is required for the perception of objects, mere spatial perception itself essentially requires the awareness of space. Of course, it will follow from AT that if Cassam's argument succeeds then it is not just 'the capacity to perceive spatial properties' but also 'the capacity to perceive space' that is a condition on perceptual knowledge. But Cassam's argument is not our concern here. 


\section{The Metaphysics of Experience}

How then is the Apriority Thesis to be argued for? According to Warren, the suppressed premise in Kant's argument appeals to the nature of geometrical knowledge: it is because '[t]he ascription of spatial relations to objects presupposes many a priori claims about what combinations of spatial relations are or are not possible' (Warren 1998, p. 207), and these claims are neither purely logical nor justifiable by mere experience, that the representation of space is required for that of spatial objects and relations. But such a premise assumes a discredited mathematical epistemology that will likely have little appeal to us, and also fails to account for the way in which Kant's claim of the apriority of space strikes us as so intuitively compelling right off the bat. A twenty-first century Apriority Theorist will wish to proceed in a different way than this.

Another aspect of what attracts Kant to AT can be found in his talk of the representation of space as a 'form of sensibility', by which he means that it 'so determines the manifold of appearance that it allows of being ordered in certain relations', and is something 'in which alone ... sensations can be posited and ordered in a certain form' (Kant 1933, p. A20/B34). To think in this way is to propose what we might call an intuitive Newtonianism, according to which the awareness of a single, overarching space serves, like physical space on Newton's view, as a sort of preexisting framework within which spatial particulars need to be placed if they are going to be perceived at all. While Cassam (2007, p. 124) complains that this way of thinking is too bound up in the peculiarities of Kantian transcendental psychology to be of much benefit to us, the general idea of an apparent structure or 'order' that must be possessed by perceptual experience in order for it to have certain further characteristics does seem natural enough; surely we can get a handle on this without taking on board Kant's psychological apparatus of sensible 
matter and form. In the present case, the thought would be that there can be no such thing as the awareness of spatial distinctness except agains the background of an overarching spatial structure: whereas I can, say, be aware of the difference in brightness between two perceived items without already employing a further perceptual representation of a 'brightness-line' that they occupy (see Warren 1998, pp. 199-204), there can be no such thing as perceiving the difference between the positions of two points without experiencing a single space within which both points are located. It thus seems impossible to perceive spatially without already being aware of space: for how could something be apparently spatial unless space itself is apparent?

Having said this, it is difficult to see how to supply a compelling argument for this conclusion except by way of something like the phenomenological method of 'free variation', that is, through exercises that attempt to determine the essential characteristics of various types of experience by exploring the limits of the imagination. As Husserl describes the procedure in Experience and Judgment:

... by an act of volition we produce free variants, each of which, just like the total process of variation itself, occurs in the subjective mode of the 'arbitrary.' It then becomes evident that a unity runs through this multiplicity of successive figures, that in such free variations of an original image, e.g., of a thing, an invariant is necessarily retained as the necessary general form, without which an object such as this thing, as an example of its kind, would not be thinkable at all. While what differentiates the variants remains indifferent to us, this form stands out in the practice of voluntary variation, and as an absolutely identical content, an invariable what, according to which all the variants coincide: a general essence. We can direct our regard toward it as toward the necessarily 
invariable, which prescribes limits to all variation practiced in the mode of the 'arbitrary,' all variation which is to be variation of the same original image, no matter how this is to be carried out. The essence proves to be that without which an object of a particular kind cannot be thought, i.e., without which the object cannot be intuitively imagined as such. (Husserl 1973, p. 341)

The phenomenologist's strategy is to draw out the essential characteristics of conscious awareness by identifying certain traits of it that remain invariant across a range of experiences that are freely generated by the imagination. And indeed Kant describes his method in the Aesthetic in terms congenial to this approach: he proposes to 'first isolate sensibility, by taking away from it everything which the understanding thinks through its concepts, so that nothing may be left save empirical intuition', and then to 'also separate off from it everything which belongs to sensation, so that nothing may remain save pure intuition and the mere form of appearances' (Kant 1933, p. A22/B36). Clearly one natural way to try to separate off the 'mere form of appearance' from the varieties of sensory matter is to see how far one's imagination can stretch the realm of possible experience, thereby revealing certain sorts of experiences - say, those of spatial properties or relations somehow independent of the awareness of space - as impossible. Though Kant never makes such a strategy explicit, it seems appropriate to draw on it in filling out his brief but intuitively compelling sketch of an argument.

Whether or not it is appropriate to read into Kant this way of arguing for the Apriority Thesis, other philosophers who have defended AT have adopted the phenomenological approach much more overtly. For example Wittgenstein, in his attempt to construct a 'phenomenological language' in the Philosophical Remarks, argues that it is 'obviously possible to establish the 
identity of a position in the visual field, since we would otherwise be unable to distinguish whether a patch always stays in the same place or whether it changes its place' (Wittgenstein 1975, pp. 253-54). And this possibility is, he goes on to claim, not one that visual experience has only contingently, but rather a condition on the possibility of visual experience in general:

If every point in visual space is marked out as distinct, then there is certainly a sense in speaking of here and there in visual space ... But is this property of having points marked out as distinct really essential to visual space; I mean, couldn't we imagine a visual space in which we could perceive only certain spatial relations but no absolute position? That is, could we picture an experience so? In something like the sense in which we can imagine the experiences of a one-eyed man? - I don't believe we could. For instance, one wouldn't be able to perceive the whole visual field turning, or rather this would be inconceivable. How would the hand of a clock look, say, when it moved around the edge of the dial? (I am imagining the sort of dial you find on many large clocks, that has only points on it, and not digits.) We would then be able to perceive the movement from one point to another - if it didn't just jump from one position to another - but once the hand had arrived at a point, we wouldn't be able to distinguish its position from the one it was in at the last point. I believe it speaks for itself that we can't visualise this. $(1975$, p. 254)

Wittgenstein's overriding concerns are different from Kant's, but clearly the two philosophers share a common conviction, namely that at least in the case of vision, there can be no experience of spatial particulars without the awareness of a space that is in some sense 'absolute', and thus that this latter sort of awareness is a condition on the possibility of the visual experience of 
spatial properties and relations, and thus also of particular spatial objects. And what Wittgenstein does that Kant does not is make it clear what this claim is supposed to follow from: it is the impossibility of imagining an experience of spatial relations without position in space that shows the impossibility of having an experience of the relevant sort.

Similarly, here is Edith Stein, in her 1917 elaboration of Husserl's notes ${ }^{1}$ on the 'Systematic Constitution of Space':

... each body must have a location in intuition; each body is, as a matter of principle, given only as oriented. 'In intuition,' we said. Then we must immediately say that each body has its location, one that belongs to it. ... The possible system of locations or system of orientations, every location of each body, is thus comparable with every other one, and therefore each body has an orientation toward all the others, a location in relation to them. (in Husserl 1997, p. 277)

Once again, we find here the idea that spatial objects cannot be perceptually presented except as positioned (that is, located and oriented) in a single space and as standing in relation to other (actual or possible) objects; there is no such thing as perceptual experience without an awareness of the twin 'systems' of location and orientation. ${ }^{2}$ And while the method of free variation had not

\footnotetext{
${ }^{1}$ The original draft text on which Stein is commenting reads as follows: '.. each body must have a location in intuition; each body is, as a matter of principle, given in intuition only as oriented. Must we not then immediately say that each body has its location, one that is given, that belongs to it? In contrast to the multiplicity of "locations," however, the body is one and the same as regards form, formed qualities, etc. The body can be completely unchanged in those respects, and if it does change, then every phase has its location and could occupy, without itself becoming a different phase, any other location of the system. The possible system of locations or system of orientations is of one type for each body, and, according to the various dimensions of the qualities having this peculiarity, every location is thus comparable with every other one, and thereby each body is also situated and oriented in relation to all the others.' (Husserl 1997, p. 257)

${ }^{2}$ In response to this gloss on Stein's view, an anonymous referee suggested that it may be wrong to run together
} 
been fully developed when Husserl gave his Ding und Raum lectures in 1907, by the time Stein expanded his notes in 1917 she would certainly have encountered his emphasis in the Ideen on the primary role of the imagination in the search for perceptual essences, which he compares to the method of geometry:

In his investigative thinking the geometer operates on the figure or model incomparably more in phantasy than in perception ... In phantasy, to be sure, he must make an effort to attain clear intuitions from which he is exempted by the sketch or model. But in actually sketching and constructing a model he is restricted; in phantasy he has incomparably more freedom reshaping at will the figures feigned, and in running through continuously modified possible shapings, thus in generating an immense number of new formations; a freedom opens up to him for the very first time an access to the expanses of essential possibilities with their infinite horizons of eidetic cognitions. (Husserl 1982, p. 159)

In the same way, Husserl continues, in investigating the essential structures of perceptual phenomena the phenomenologist 'can use the resource of ordinary givenness only to a limited extent', as 'he does not have examples for all possible particular formations any more than the geometer has sketches or models at his disposal for the infinitely many kinds of solids.' As in geometry, 'the freedom of eidetic research ... necessarily demands operating in phantasy' (ibid.). It is not a long way from these ideas to the sorts of imaginative exercises proposed to us by

the perception of orientation and location as Stein seems to: e.g., can't we perceive a star as located in space without its having any orientation? But Stein may be thinking also of the sort of thing Wittgenstein refers to with his talk of 'absolute position' in the passage quoted above: that is, the fact that perceptual space itself has an apparent orientation, a 'top' and 'bottom' and 'left' and 'right' according to which perceived locations are defined. I have more to say about this idea in section 4.2 below. 
Wittgenstein: it is because a perception of spatial objects, properties, and relations independent of the awareness of space itself is found to be unimaginable that we are entitled to regard it an experiential impossibility.

With this argumentative strategy in mind, it is worth returning to an aspect of Wittgenstein's argument that we mentioned only in passing, namely the restriction of its conclusion to the domain of visual experience. Given what we have just said, it is easy to see the motivation for such a restriction: for along with touch vision is the paradigmatically spatial modality of perceptual awareness, and unlike touch vision clearly presents a range of spatial locations in a 'simultaneous' fashion, and not just 'successively' one after the other. For this reason, the conclusion of the imaginative exercise Wittgenstein invites us to conduct is especially easy to reach when we restrict our attention to vision; it is somewhat harder to argue in this way that there must be such a thing as an absolute 'here' and 'there' in the space that is characteristic of, say, audition, simply because we have a far less vivid awareness of auditory space. Thus since my stance toward the Apriority Thesis in this paper is going to be largely critical, I am going to confine my attention to versions of it that are concerned only with visual experience, assuming that if such a restricted version of AT can be brought into question, then the case for its truth in other sensory domains will have been undermined as well. It seems fair to focus our critical attention on the place where the position we are investigating is intuitively at its strongest.

\section{When Visual Space Goes Missing: A Case Study}

We are considering the following thesis: 
(AT) At least in the domain of visual experience, it is impossible for there to be spatial perception (i.e., perception of spatial objects, properties, and relations) without the awareness of space (i.e., 'space in the strict sense', or an overarching and somehow 'absolute' space within which everything is perceived as situated).

Behind the Apriority Thesis is an appeal to the limits of the imaginative variation of visual possibilities, which generates a conclusion concerning the essential character of visual experience in general. This argument is, of course, distinctively philosophical: it advances claims about the necessary structure of conscious experience in ways that make no appeal to laboratory experiment or other narrowly empirical considerations. And clearly this style of argument can become dangerous if it is extended beyond the metaphysics of experience, and made to apply to the structure of the physical universe itself: for without a lot of dubious metaphysical theorizing of the sort we find in Kant, we will have little good reason to claim that the necessary structures of the universe must reflect the way that we human subjects are constrained to perceive or otherwise represent it. But our way of interpreting the Apriority Thesis avoids this problem by limiting its application just to our particular kinds of minds, proposing that our capacity for imagination gives us a kind of intimate access to the nature of perceptual experience that permits us to lay bare its essential characteristics purely from the philosophical armchair.

This does not mean, however, that the Apriority Theorist is entitled to regard empirical data as altogether irrelevant to the evaluation of his or her view. For even if claims about metaphysical necessities cannot reasonably be supported by a posteriori evidence, they nevertheless yield empirical predictions, and can therefore be put to the test by considering whether those predictions hold up. As José Luis Bermúdez has put it: 
Neuropsychology can bring ... thought-experiments to life. It allows the formulation and testing of predictions. If a transcendental argument claims that the possibility of a subject's having a particular conceptual ability A is dependent upon his possessing a further conceptual ability B, then this clearly generates the prediction that A cannot exist in the absence of B. We can test such predictions by looking at what happens in neuropathies where ability B is severely impaired. If the argument is sound, we would expect the patient also to lack ability A. If, however, ability A remains unimpaired, then clearly the argument needs further examination. (Bermúdez 1995, pp. 381-82)

In the case of the Apriority Thesis, the relevant prediction will be that since the perception of spatial objects, properties, and relations requires the awareness of space in the strict sense, any individual in whom the capacity for the latter sort of awareness is extinguished or impaired should be correspondingly deficient in the capacity for 'mere' spatial perception. Since the Apriority Thesis asserts a metaphysical necessity, clearly its proponents cannot find real support for their view in particular cases where this relationship is present; but by the same token, they will clearly be in trouble if there turn out to be cases in which it is not.

With this in mind, consider patient 'RM', who was studied for a number of years by Lynn Robertson and her colleagues. RM had suffered a pair of strokes that resulted in severe damage to his posterior parietal cortex, and as a consequence was unable to localize the objects he saw. For example, when shown a display with a target at one of five locations along the vertical or horizontal meridians and told to report whether the target's position was up, down, or center (in the vertical blocks) or right, left, or center (in the horizontal ones), RM averaged only $70 \%$ 
correct across all conditions. Similarly, when instructed to judge the relative position - left or right in one block, up or down in the other - of an ' $\mathrm{X}$ ' with respect to that of an ' $\mathrm{O}$ ' that was also presented on the screen, RM was correct approximately $50 \%$ of the time, a performance no better than chance (Friedman-Hill, Robertson, and Treisman 1995). RM could detect the target stimuli well enough; he just couldn't tell where they were, either on the screen itself or with respect to other things.

Significantly for our purposes, RM's deficits in visually-based localization were not a product of straightforward misperception, as if he routinely experienced stimuli as being to the top of the screen when they were in fact at the bottom, and so on. Rather, when instructed to report on the location of a visually presented object RM 'had to be prodded to guess', and 'would shake his head back and forth and protest that he did not know where the word was' (Robertson 2004, pp. 170). As Robertson puts it:

During early testing of his extrapersonal spatial abilities he often made statements like, 'See, that's my problem. I can't see where it is.' He also found it hard to describe what his perception was like. His explanations suggested that objects that popped into his view were not mislocated per se. Rather, they simply had no location in his perceptual experience. (2004, pp. 158-59)

So on RM's own account, he (1) could see only one item at a time, and (2) seems to have experienced those things as having no visually apparent location or orientation whatsoever. This seems as good a test case as any for the sorts of conclusions advanced by the Apriority Theorists so far canvassed: for it was precisely the capacity visually to experience particular things as 
located and oriented in space that RM's neurological condition seems to have extinguished. Thus the crucial question for the Apriority Theorist is whether this impaired visual awareness of space was accompanied by corresponding deficiencies in RM's visual perception of spatial objects, properties, and relations.

\subsection{A Causal Role for Spatial Awareness?}

A recent paper by John Campbell interprets the case of RM in a way that would seem to confirm at least some of the Apriority Theorist's predictions, arguing that RM had 'impaired object perception' (Campbell 2007, p. 550), and that we can attribute this impairment to his visual spatial deficits. The key piece of evidence that Campbell cites for his view concerns RM's relative inability to detect stimuli whose status as targets was defined by the possession of conjunctions of properties along different featural dimensions. For instance, when presented with displays consisting of two colored letters and asked to report the name and color of the first letter he saw, RM reported 'illusory conjunctions' - in which the color of one object was experienced as conjoined with the shape of the other - at least 13 percent of the time, even with display times of as long as ten seconds (Friedman-Hill, Robertson, and Treisman 1995). He seems to have perceived the features (in this case, the colors and the shapes) that were on the screen while misperceiving the way those features were components of unitary objects. And since, according to Campbell, to perceive an object is to perceive 'a single thing with many properties' (Campbell 2007, p. 550), he concludes that RM's impaired capacity for visual spatial awareness causally affected his capacity for visual object perception.

Following Robertson, Campbell proposes that we can get an initial handle on why there might be a causal relationship between spatial awareness and object perception in terms of the 
notion of a visual 'master map', as it figures in Anne Treisman's Feature Integration Theory of visual attention (Treisman and Gelade 1980). According to this well-known approach to the visual binding problem, different sorts of visible features - colors, shapes, orientations, and so on - are detected by the human visual system in an early and automatic fashion and represented in distinct visual subsystems, each with its own rough map of where those properties were detected. Visual attention, by 'spotlighting' a particular location in visual space, then leads the visual system to check all the various feature maps and determine which properties were perceived at that location, thereby recombining them into a unitary visual representation of an object.

There is considerable evidence available to support such a view, one instance of which will suffice for our purposes. Suppose you are asked to report the presence of a single 'target' stimulus from within an array of distractors: FIT predicts that if the status of the target is defined by a single property (such as color or shape alone), then automatic 'feature-detection' will suffice, and you will be able to detect the target immediately and so without interference from the total number of non-target elements. If, on the other hand, the target is defined as a conjunction of two or more distinct kinds of properties (a red ' $\mathrm{X}$ ', say), then focal attention to its location will be required to detect it, and response times will increase linearly with the number of objects to be scanned. And in normal subjects, this is exactly what we find.

Crucially for the present discussion, Campbell builds on this theory by proposing that it is specifically the conscious visual awareness of location that is required for visual feature-binding to occur, as RM's visual system appears to have represented information about spatial location below the level of consciousness. For example, when RM was shown a display consisting of a vertically oriented rectangle with one of the words 'UP' or 'DOWN' presented at its top or bottom and asked to (1) read the word and (2) report which end of the rectangle it was located in, 
his responses exhibited the Stroop effect (Stroop 1935): despite being unable to tell where the words were located, RM was significantly slower to report the identity of the word in the 'incompatible' conditions (where 'UP' was at the bottom of the rectangle, or 'DOWN' at the top) than the 'compatible' ones (Robertson, Treisman, Friedman-Hill, and Grabowecky 1997). ${ }^{3}$ This shows that the locations of the words with respect to the rectangles were represented somewhere in RM's visual system, and that this information affected his verbal reports. Nonetheless, he clearly was not aware - consciously, that is - of the words' locations: as noted above, when asked to report where they were RM insisted that he did not know, and 'had to be prodded to guess the location' (Robertson 2004, p. 170); and when he did come to hazard a guess, it was accurate only $51 \%$ of the time (at chance, for a two-alternative forced-choice task). RM's intact 'implicit' visual representation of spatial information - as opposed to the 'explicit' grasp of it that would have come with conscious visual awareness of spatial location - was not enough on its own to enable the veridical binding of perceived properties into unitary representations of individual objects. ${ }^{4}$ Thus, Campbell concludes, deficits in visual spatial awareness make for deficits in the visual perception of objects.

But in fact things are not so straightforward. For notwithstanding the perceptual deficits that Campbell discusses, RM seems to have been perfectly capable of another type of conscious visual spatial awareness, as illustrated for example by the results of a simple letter identification task. Here, he was simply shown a single letter, and asked to report what he saw. In a block in

\footnotetext{
${ }^{3}$ Relatedly, Wojciulik and Kanwisher (1998) report that RM exhibited a similar effect in tasks requiring feature binding (e.g. reading a color-word that itself was printed in a compatible or incompatible color), suggesting that he had some implicit access to featural conjunctions that did not rise to conscious awareness. I am grateful to an anonymous referee for calling this study to my attention.

${ }^{4}$ To say this is not, however, to foreclose the possibility that more careful empirical investigation might reveal the possibility of a 'blindsighted' solution to the binding problem, by turning up subjects in whom the awareness of location is impaired but who can nevertheless detect conjunctions of features with a high rate of success. (Here I am grateful to Erika Klempner.)
} 
which the stimuli consisted of the letters 'b', 'd', 'p', and 'q', RM identified the letter correctly on 23 of 32 trials, and five of his nine errors consisted in confusing mirror-image pairs.

Similarly, in a block whose stimuli were the letters 'o', 'e', 'c', and 'd', RM made only one error in 16 trials, and that was to read the 'd' as a 'b', another case of mere mirror inversion (Robertson \& Treisman 2006). What is remarkable about this finding is that, just as in the studies described earlier that tested the awareness of location or the binding of features into wholes, RM clearly had accurate visual experiences of the intrinsic shapes of the target objects, and so was able to see how their proper parts fitted together to form spatial wholes. He did not, for example, experience the straight line in a ' $\mathrm{d}$ ' as extending one side of the curve, as in a capital ' $\mathrm{J}$ ', or the sideways line in an 'e' as running diagonally through the curve, as in a ' $\varnothing$ ' sign; rather, his visual errors were limited to illicit rotations and mirror-image reflections. Up to a point, then, his visual spatial awareness was perfectly intact. RM was entirely able to see the intrinsic spatial form of a particular figure, even as he lacked any visual awareness of how such figures were located and oriented in space itself.

So it is important not to overstate the extent of RM's visuospatial impairments (as Campbell seems to, when he says for instance that RM 'had no spatial awareness' (Campbell 2007, p. 550); compare this to Robertson's somewhat more cautious description of his condition as one in which there was 'no "there" there' (Robertson 2004, p. 6)). As Robertson and her colleagues put it, it was RM's visual experience of extrinsic or between-object spatial properties and relations that had gone awry, while that of intrinsic or within-object spatial features was largely intact in his case. This is why he could perceive the intrinsic shapes of the letters accurately up to isomorphism, but had nothing to say when asked to rely on a sense of how things were positioned in space. The visual awareness of space seems to have two distinct 
'dimensions', as it were, only one of which is implicated in binding features such as shape and color together in the representation of a unified object.

Before turning to the question of how the case of RM bears on the argument of the Apriority Theorist, it will be worth dwelling a bit longer on the way in which the distinction between intrinsic and extrinsic spatial features challenges Campbell's diagnosis of RM's condition. Recall that according to Campbell RM's visuospatial deficits led to corresponding deficits in his capacity for visual object perception, where this is defined as the perception of 'a single thing with many properties'. By contrast, consider the following quote from Robertson and her colleagues, in which the capacity to conjoin different kinds of features into a veridical representation of an object is treated as less essential to what they call 'object perception' than the awareness of intrinsic shape itself:

Patients with Balint's syndrome can identify a perceived object. That is, they are able to perceive a defining shape. Because of this ability, it was assumed that they saw the object with all its features intact, but this turns out not to be the case. The assumption also led to the proposition that spatial attention was reduced in those patients to cover only the spatial extent of the object perceived. However, this does not capture the full dimensions of the problem either. We have shown that such patients have abnormal feature binding as well. They miscombine a feature such as colour, size, or motion in the scene with the shape they perceive. (Friedman-Hill, Robertson, Desimone, and Ungerleider 2003, p. 424; emphasis added)

In this quotation object perception seems to be regarded as one thing, and veridical feature 
binding as quite another. The former is supposed to rest crucially on the veridical experience of the intrinsic shape of an object, while the latter involves attaching further properties to an object that has - in virtue of the successful recognition of its shape - already been perceived. And on this understanding, the visual awareness of space in the strict sense seems to be causally irrelevant in visual object perception, since as we have already seen the perception of a thing's 'defining shape' does not require conscious awareness of its location.

This latter way of understanding what is required for object perception is the one which, by and large, Robertson and her colleagues rely on in describing RM's condition. They say, for example, that he 'is frequently aware of only one object in the visual field or one group of clustered objects' (Friedman-Hill, Robertson, Desimone, and Ungerleider 2003, p. 4264); and similarly, they write in another paper that 'When shown two objects he often only saw one. When he did report both, he did so slowly, and seemed to see them sequentially' (Robertson, Treisman, Friedman-Hill, and Grabowecky 1997, p. 297). These quotations are by no means uncharacteristic; in general, RM and other Bálint's patients are consistently described in the neurological literature as individuals who perceive objects but are unaware of their locations and sometimes misperceive certain of their features.

We can find some philosophical support for this way of thinking in the idea that the defining shape of a physical object occupies a special status among the properties that make it the kind of entity it is: in the same way that, as Cassam puts it, 'the capacity to perceive their primary qualities is ... a necessary condition for the perception of material objects because it is in virtue of their primary qualities that they count as material objects in the first place' (Cassam 2007, p. 124), so the perception of some important subset of an object's primary qualities may also be sufficient to perceive that object. This line of argument is further strengthened when we 
consider that not only is an object's defining shape a primary quality, but it is also a property intrinsic to the object that has it: that is, it is an aspect of the object that it possesses independently of its relations to anything else, including (if indeed it makes sense to think this way) the larger space that surrounds it. Thinking about material objects in this way, and compiling for argument's sake the union of all of Locke's various lists of primary qualities (see Alexander 1985, pp. 133-34), we can propose that among solidity, bulk, figure, mobility, situation, number, texture, and motion of parts, it is because figure is both (i) a primary quality that (ii) is intrinsic to the thing whose figure it is, that the perception of an object's defining shape suffices for the perception of that object itself.

If this is the criterion for visual object perception that we adopt, then Campbell's conclusion rests on a misdiagnosis of what has gone wrong in RM: as Robinson and her colleagues suggest, it is not that his capacity visually to perceive objects that was affected by his impaired visual awareness of space, but rather his capacity for the veridical visual perception of certain of the properties that those perceived objects have. But as we have seen, according to Campbell his stronger conclusion is warranted because object perception requires more than the perception of mere features; rather, it further requires the perception of how those features are combined into single things. As he puts it:

His [RM's] object perception was impaired, in the following sense. He could not identify which conjunctions of features were present in an array: for example, he could not tell whether a red square was present in an array of variously colored circles and squares. Nonetheless, he was relatively good, well above chance, at saying which features were present in an array, even though he could not reliably say which features were conjoined 
with which. So he could tell whether redness was present and whether squareness was present, even though he could not reliably tell whether a red square was present. If we think of an object as a single thing with many properties, we can describe the situation by saying that RM had impaired object perception but intact feature perception. (Campbell 2007, p. 550; emphasis added)

Yet Campbell can describe things in this way only by insisting that the visual perception of a figure's intrinsic shape does not already qualify as the perception of 'a single thing with many properties', as opposed to a mere bunch of features present in an array. And there is good reason to reject this claim: for example, in the letter identification task described above where RM correctly identified a lowercase 'e', it would have been impossible for him to do this had he not perceived the very same object both as having a curved side and also as having a horizontal line through the middle. Even if there were others of the figure's properties - its size, its location, its orientation, and perhaps also its color - that he failed to perceive, the content of his visual experience still involved something analogous to the ascription of multiple predicates to a singular referent: he perceived a particular thing as having a complex shape involving multiple figural aspects. (Indeed, Baylis et al (1994) found that RM could identify words of up to four letters, both reading them as wholes and identifying the letters they were composed of. ${ }^{5}$ ) And so it seems that we should say that RM satisfied even Campbell's own requirements for visual object perception, as the mere perception of the structurally complex shape of a thing suffices for the perception of that object itself.

Importantly, this sort of featural complexity is a very general characteristic of the way

\footnotetext{
${ }^{5}$ Thanks to an anonymous referee for calling this study to my attention.
} 
intrinsic shapes are presented in visual experience, and the idea that shape perception requires a kind of binding together of visible properties is an important part of the psychological literature. For example, Treisman distinguishes seven different types of binding relevant to visual object perception, among them part binding, in which 'the parts of the object [are] segregated from the background, and bound together' (Treisman 1996, p. 171). That RM consistently perceived the intrinsic shapes of letters and even words clearly suggests that there is a kind of visual binding the product of which, even on Campbell's own terms, deserves to be called a kind of object perception, and which was perfectly intact despite his visuospatial deficits. In any case, it seems wrong to say that all RM was able to do visually was to 'say which features were present in an array': he could do this, but of some of these features he could also tell 'which features were conjoined with which', and moreover how those features fit together into unitary spatial wholes. We should reject the claim that RM's visual impairments left him unable visually to perceive objects. His condition ultimately contradicts, rather than supporting, the idea that the visual awareness of space is a precondition on the visual perception of objects.

\subsection{Does RM's Condition Falsify the Apriority Thesis?}

While seeing the mistaken in Campbell's diagnosis of RM's condition requires this detour through the concept of object perception, the intuitive difficulty that RM raises for the Apriority Thesis is much more straightforward. For according to the explication we gave above, the Apriority Theorist's working picture is of visual space as an overarching framework which must be 'in place' so that spatial objects, properties, and relations can be experienced within it. In RM's condition, however, it was precisely an aspect of 'mere' spatial perception, i.e. the visual awareness of the intrinsic spatial form of particular physical objects, that remained entirely intact 
despite his incapacity for visual spatial awareness in the strict sense, i.e. of such objects and their intrinsic spatial properties as positioned within a larger space. That is, according to Kant 'the representation of space must be presupposed' in order for sensations to be represented 'as outside and alongside one another, and accordingly as not only different but as in different places' - yet RM's intact visual awareness of the shapes of things constitutes a representation of the latter sort, achieved without representing space itself. Whereas AT sees the awareness of within-object spatial relations as a special case of the awareness of the spatial relations that hold between distinct things, and so the experience of shapes and spatial properties and relations as derivative upon what we have called the experience of space in the strict sense, RM's condition indicates that in the visual domain, these perceptual achievements can be entirely distinct. ${ }^{6}$

Given RM's insistence that he did not experience objects as having locations within any sort of larger space, the only way for an Apriority Theorist to account for his condition without denying his introspective judgments is by arguing that his awareness of particular shapes itself amounted to a way of being aware of space in what we have called 'the strict sense'. The idea here would be that the awareness of the intrinsic shape of a figure involves the awareness not just of spatial properties or relations but also of the bounded region of space that that figure takes up - an 'object-space', as we might put it. On this analysis of RM's condition, his simultanagnosia saddled him with radical sort of tunnel vision, ${ }^{7}$ where there was no more space visible to him than that which a perceived object occupied; however, this should not be equated with his not having been aware of space at all.

\footnotetext{
${ }^{6}$ In comments on an earlier version of this paper, an anonymous referee pointed out that since RM's condition was acquired, it doesn't rule out the possibility that some past capacity for the awareness of space may have been required for spatial perception. But of course the Apriority Thesis says something other than this; and moreover it is hard to see how this alternative hypothesis could be argued for in the way we have been considering.

${ }^{7}$ Here I am indebted to a helpful conversation with Mike Martin.
} 
Yet we should take care in advancing such a diagnosis. Recall Wittgenstein's discussion of the moving hand on the face of a clock, or the possibility of experiencing the rotation of the entire visual field: what these examples are supposed to show is that visual space involves something more than just the intrinsic spatial structure of a particular object; it also involves the awareness of such an object as positioned in space. And to the extent that we can imagine the possibility of tunnel vision, this is precisely how it strikes us: we imagine experiencing an object, and in that experience one side of that object is at the top of the visual field, another at the bottom, and so on. As Wittgenstein puts it, visual space must be 'an oriented space, a space in which there is an above and below and a right and left' (Wittgenstein 1975, p. 255). So if the Apriority Theorist is to claim that RM was aware not only of shapes but also of the 'objectspaces' that those shapes took up, then it must be shown that the shapes he perceived were experienced as 'oriented' in this way.

To support this interpretation, Apriority Theorist might note that in the letteridentification tasks discussed above, RM was significantly above chance not only in identifying the intrinsic shapes of the letters, but also in discriminating between mirror-image pairs. And there is a Kantian line of thought which holds that the ability to do this sort of thing requires experiencing things as positioned within an 'absolute' space:

What indeed can be more similar to, and in all parts more equal to, my hand or my ear than its image in the mirror? And yet I cannot put such a hand as is seen in the mirror in the place of its original; for if the one was a right hand, then the other in the mirror is a left, and the image of the right ear is a left one, which can never take the place of the former. Now there are no differences hear that any understanding can merely think; and 
yet the differences are inner as far as the senses teach ... What then is the solution? ... space is the form of outer intuition of [our] sensibility, and the inner determination of any space is possible only through the determination of the outer relation to the whole space of which the space is a part ...; that is, the part is possible only through the whole ... (Kant 1997, p. 38)

If what Kant says here is right, then it follows that RM's visual sensitivity to the differences between 'incongruent counterparts' like a lowercase 'p' and a lowercase ' $q$ ' shows that he did, after all, experience those objects as positioned in space, for there is nothing else that could account for the visual difference between them. On this analysis, the strangest thing about RM's condition would be only that visual space had 'shrunk' in his case to the size of whichever single object he saw.

But Kant's position elides an important distinction between two different ways of relating an object to a spatial frame of reference. On the one hand, an object may be represented, perceptually or otherwise, with respect to a 'global' reference frame, which determines its position and orientation in space and its relations to other actual or possible spatial objects; and on the other hand, the intrinsic spatial structure of an object can be represented according to its own 'object-centered' frame of reference, according to which the relations between its own proper parts are determined. It is this distinction that accounts, for example, for the aspects of constancy in the visible appearance of an object even as it is seen to rotate or move around in space: the relation of the object to its object-centered frame remains unchanged, while its position according to the global frame varies. Relating the parts of an object to an objectcentered frame ('This is the top of the thing, and this is the bottom, etc.') is not just a matter of 
relating those parts 'to the whole space of which the space [taken up by the object] is a part'; for if it were, it is hard to see how it would be possible, say, to experience the top of a thing as down, as for example when you see a gymnast standing on her head while the floor looks to be below her. And there are compelling reasons to think that RM could represent spatial relations only according to object-centered frames of reference, as for example in an experiment where Robinson and her colleagues showed him the words 'NO' and 'ON' and asked him to report whether the letter ' $N$ ' was on the left or the right:

He found the task very difficult, took a long time to respond, and was at chance performance. However, when we asked him to read the word NO or ON in other blocks of trials he was $69 \%$ accurate (clearly not good, but significantly better than when his task was to locate the $\mathrm{N}$ ). Although he could not explicitly access the location, there was evidence of some implicit encoding of spatial information that influenced the identification of the word. (Robertson 2004, p. 180)

As Robertson suggests, RM's ability to distinguish between these mirror-image pairs is evidence that his visual system registered the letters' positions with respect to a global frame of reference defined by the space of the display. But the fact that he could not report those positions clearly indicates that he was not explicitly aware of them as such: rather, his implicit sensitivity to the letters' positions on the global frame led his visual system to assign an object-centered frame to each stimulus, defining one side as the top of the word, another side as its bottom, and so on. That is, he perceived each word as having an intrinsic spatial structure that determined its identity, but not as having any determinate orientation in visual space. 
Robertson and her colleagues found further evidence for this interpretation when they repeated this test with strings of letters that had no intrinsic semantic significance:

$\ldots$ we replaced the words $\mathrm{ON}$ and $\mathrm{NO}$ with the less frequent letter strings $\mathrm{OZ}$ and $\mathrm{ZO}$. We hypothesized that this would decrease the influence of top-down mechanisms because the semantics of these letter strings would not be accessed automatically. As predicted, RM was no better than chance at either reading the word or localizing one of its letters when the letter strings were less familiar. ... these findings suggest that the more familiar the word was the more top-down information influenced his ability to perceive the word as its proper whole. (ibid.)

This last phrase is crucial: for as we have already noted, RM's capacity to perceive an object 'as its proper whole' clearly amounted to a kind of spatial perception; and moreover his capacity to distinguish between mirror-image pairs in certain cases required a visual sensitivity to extrinsic spatial properties in addition to intrinsic ones. But visually experiencing the difference between mirror-image pairs is not simply a matter of representing where a thing lies according to a global frame of reference: for once again, if this were so then there would be no way for, say, the word 'NO' to be turned on its head without its appearance simply morphing into that of the word 'ON'. (If you doubt whether this is possible, turn your paper upside-down and read the following sentence: 'THERE IS NO ONE HERE, EXCEPT ON TUESDAY'.) And moreover, what the case of RM shows is that in certain cases it is possible to perceive the differences between such pairs without experiencing them as having spatial orientations at all: thus for RM there will be no possibility, for example, of the rotation of the entire visual field, as opposed to the 
replacement of an object whose intrinsic spatial structure is defined by a certain object-centered frame with an object that is structurally similar but whose intrinsic spatial properties are different, as when the letter ' $\mathrm{N}$ ' simply transforms into a ' $\mathrm{Z}$ '. This is not mere tunnel vision; indeed, it is not the sort of thing that we can picture to ourselves at all.

To sum this up: if it were true that RM experienced the 'object-space' taken up by an object whose shape he saw, then he would have experienced that object not just as having a top and a bottom and a left and a right, but also as oriented, albeit in a region of space fully taken up by it. Kant's argument from incongruent counterparts concludes that this cannot have failed to be so, but does so at the expense of a phenomenologically and empirically important distinction. And what evidence there is concerning RM's capacity for shape perception clearly suggests that his experience was of a wholly unimaginable sort: not of oriented spaces that were cut off at the boundaries of the things that occupied them, but of things without spatial locations or orientations. RM perceived shapes that did not appear to be in space at all. ${ }^{8}$

\footnotetext{
${ }^{8}$ A quick note on how the failure of the Apriority Thesis bears on the Leibnizian account of spatial representation that is one of Kant's primary targets in the Metaphysical Exposition and the argument from incongruent counterparts. According to Leibniz in $\$ 47$ of his Fifth Letter to Clarke (Leibniz and Clarke 2000, pp. 45-47), the concept of space is formed by 'assembling', as it were, the concepts of particular spaces, which in turn are formed from the concepts of spatial properties and relations. Kant's arguments for the priority of the representation of space to mere spatial representation are supposed to show that such a procedure would be impossible; but it is important to see that the choice between AT and the Leibnizian view is not exhaustive. For it could be that even though the representation of space is not positively required for the representation of spatial properties and relations, nevertheless the former representation is sui generis in a way that rules out its being formed solely on the basis of these others. Indeed, it is possible to interpret the case of RM in a way that supports this position, though Bill Prinzmetal has convinced me that a 'Leibnizian' account of his condition is also compatible
} 


\section{Methodological Observations}

What does this discussion suggest about the philosophical value of the sort of a priori reflection that motivates the Apriority Thesis? Cassam glosses what he calls explanatory minimalism as the denial 'that there are substantive enabling conditions for the acquisition of perceptual knowledge that, unlike physiological and biological conditions, can be established without any empirical investigation' (Cassam 2007, p. 87). Clearly the Apriority Thesis proposes a condition on the possibility of perceptual experience of exactly the sort that a minimalist will reject out of hand: so does the fact that we seem to have proven it wrong by appealing to empirical considerations provide any support for a minimalist position more generally? In the face of these results, what are we to think of the value of a priori inquiry into the nature of the mind? Must we abandon hope for fruitful phenomenological investigation of the necessary structures of consciousness and human knowledge, instead ceding these subjects entirely to experimental science?

Across the philosophical spectrum from the minimalist's radical empiricism we can juxtapose a staunch conservatism about the conclusions of a priori investigation, according to which they should be upheld in the face of a posteriori scrutiny almost come what may. On this view the positions we arrive at by way of phenomenological inquiry and other modes of armchair analysis do indeed generate empirical predictions, but it is the responsibility of the philosopher-scientist to reinterpret initially recalcitrant empirical data to bring them into line with common sense. Thus a conservative who is deeply persuaded by the kinds of

with the available data. Going further into the details of this would take us too far afield. I am grateful to Bill Prinzmetal, Lynn Robertson, Angela Schwenkler, and Daniel Warren for helping me think through these issues. 
phenomenological analyses described in section 3 might argue that our inability to imagine visual experiences anything like those that RM claims to have had - e.g., ones in which visible objects 'simply had no location in his perceptual experience', or ones in which intrinsic spatial structure was experienced but 'absolute' spatial orientation was not - gives us license to be skeptical of RM's ability accurately to describe what his visual experience was like: perhaps he couldn't report on where things visually appeared to him to be, but that doesn't mean that there wasn't a fact of the matter about how they were positioned in his visual field. Alternatively, a conservative might push back against the idea that RM really enjoyed conscious visual experiences of the intrinsic shapes of things after all. ${ }^{9}$ On this view, the subjective reports of someone like RM should not undermine our considered philosophical convictions about the scope of experiential possibility.

There are many things that can be said in response to this sort of position, but two points seem especially apposite. The first is that, conceding for a moment that conservatism may be an acceptable position in general, in this particular case it is not as if the conceptual distinction between the visual awareness of space and that of particular shapes and spatial properties has no echo at all in our visual phenomenology: as we noted just above, you can for example experience the very same object first as upright and then as lying on its side, but without experiencing any change at all in the intrinsic shape it looks to have. This clearly indicates that the visual awareness of within- and between-object spatial properties cannot simply be reduced to visual spatial awareness of a single basic sort, as an especially clumsy version of the Apriority Thesis might have it; and while this observation certainly does not entail that these two aspects of visual experience can be teased entirely apart from one another, it at least provides an initial

\footnotetext{
${ }^{9}$ I am grateful to Daniel Warren and Alva Noë for pushing me to take this sort of position more seriously.
} 
experiential analogue of the independence claim that our investigation of RM's condition led us to draw.

Secondly and more generally, there is good reason to resist the idea that the limits of the imagination are a perfect guide even to the limits of possible experience: to cite just one example, a sufficiently ignorant and unimaginative person raised in a thoroughly grayscale environment may find it inconceivable - may find herself unable to 'picture' the possibility that a person might enjoy a huge range of visual experiences of a sort hitherto unknown to her. Thus in the same way that, say, mathematicians' postulations of non-Euclidean spaces forces a revision in our intuitive understanding of the conditions under which lines can and cannot intersect, so the conditions of individuals like RM should challenge us to reconsider even our most basic phenomenological intuitions, by presenting us with human subjects who, though like us in a lot of important respects, have minds that differ in significant ways from our own. Of course we should be cautious in the way we interpret their subjective reports and draw out their philosophical consequences, but in general it seems wise to appeal to further empirical investigation, rather than more first-personal phenomenology coming from our own side, if we want to claim that what such individuals say about their own experiences cannot be right. And with RM, whose verbal spatial vocabulary and capacities for spatial reasoning and spatial perception in non-visual modalities were perfectly intact, there is little independent reason to doubt what he says about the nature of his experience: his claims about what visual experience is like in his own case seem to be on stronger ground than even our most philosophically reflective armchair intuitions about what is and is not visually possible.

But this is not to concede the dialectical ground entirely to the minimalist, and so to reject altogether the endeavor to seek substantive knowledge of the essential structures of the mind 
through non-empirical means. For it is still open to us to hold that phenomenological exercises and other sorts of a priori philosophical investigation can provide a kind of evidence for claims about, say, what visual experience must be like even if that evidence is not, as conservatives like Kant and the classical phenomenologists would have had it, entirely incontrovertible and so immune to empirical scrutiny. What the case of RM should teach us is not to give up altogether on the possibility of substantive non-empirical knowledge about the nature of the mind, but rather to treat the apparent fruits of our phenomenological inquiries just as Quine taught us to treat any other putative instances of a priori knowledge: they may well warrant a place somewhere near the center of our web of belief, but are not thereby immune to revision in the light of what we learn down the line. It is by being bold in articulating the empirical phenomena that their a priori conclusions require them to predict, and meticulous in seeking out and scrutinizing findings that can disconfirm them, that practitioners of non-empirical methodologies can make genuine contributions to the study of the mind.

Department of Philosophy

Mount St. Mary's University

\section{References}

Alexander, P. 1985: Ideas, Qualities, and Corpuscles: Locke and Boyle on the External World. New York: Cambridge University Press.

Baylis, G.C., Driver, J., Baylis, L.L., and Rafal, R.D. 1994: Perception of letters and words in Balint's syndrome: evidence for the unity of words. Neuropsychologia, 32, 1273-86.

Bermúdez, J. L. 1995: Transcendental arguments and psychology: the example of 
O'Shaughnessy on intentional action. Metaphilosophy, 26, 379-91.

Campbell, J. J. 1993: Past, Space, and Self. Cambridge: The MIT Press.

Campbell, J. 2007: What's the role of spatial awareness in visual perception of objects? Mind and Language, 22, 548-62.

Cassam, Q. 2007: The Possibility of Knowledge. New York: Oxford University Press.

Freidman-Hill, S., Robertson, L.C., Desimone, R., and Ungerleider, L. G. 2003: Posterior parietal cortex and the filtering of distractors. Proceedings of the National Academy of Sciences, 100, 4263-68.

Friedman-Hill, S., Robertson, L., and Treisman, A. 1995: Parietal contributions to visual featurebinding: evidence from a patient with bilateral lesions. Science, 269, 853-55.

Guyer, P. 1987: Kant and the Claims of Knowledge. New York: Cambridge University Press.

Husserl, E. 1973: Experience and Judgment: Investigations in a Genealogy of Logic. Trans. J. S. Churchill and K. Ameriks. Evanston, Ill.: Northwestern University Press.

Husserl, E. 1982: Ideas Pertaining to a Pure Phenomenology and to a Phenomenological Philosophy. First Book: General Introduction to a Pure Phenomenology. Trans. F. Kersten. Boston: Martinus Nijhoff.

Husserl, E. 1997: Thing and Space: Lectures of 1907. Trans. R. Rojcewicz. Dordrecht: Kluwer.

Kant, I. 1933: Critique of Pure Reason. Trans. N. Kemp Smith. New York: Palgrave Macmillan.

Kant, I. 1997: Prolegomena to Any Future Metaphysics. Trans. G. Hatfield. New York: Cambridge University Press.

Leibniz, G.W. and Clarke, S. 2000: Correspondence. Ed. R. Ariew. Indianapolis: Hackett 


\section{Publishing Company.}

Robertson, L. 2004: Space, Objects, Minds, and Brains. New York: Psychology Press.

Robertson, L. and Treisman, A. 2006: Attending to space within and between objects: implications from a patient with Balint's syndrome. Cognitive Neuropsychology, 23, 448-62.

Robertson, L., Treisman, A., Friedman-Hill, S., and Grabowecky, M. 1997: The interaction of spatial and object pathways: evidence from Balint's syndrome. Journal of Cognitive Neuroscience, 9, 295-317.

Stroop, J. R. 1935: Studies of interference in serial verbal reactions. Journal of Experimental Psychology, 18, 643-62.

Treisman, A. 1988: Features and objects: the fourteenth Bartlett Memorial Lecture. The Quarterly Journal of Experimental Psychology Section A, 40, 201-37.

Treisman, A. 1996: The binding problem. Current Opinion in Neurobiology, 6, 171-78.

Treisman, A. and Gelade, G. 1980: A feature-integration theory of attention. Cognitive Psychology, 12, 97-136.

Warren, D. 1998: Kant and the apriority of space. Philosophical Review, 107, 179-224.

Wittgenstein, L. 1975: Philosophical Remarks. Trans. R. Hargreaves and R. White. Chicago: University of Chicago Press.

Wojciulik, W., and Kanwisher, M. 1998: Implicit but not explicit feature binding in a Balint's patient. Visual Cognition, 5, 157-82. 